\title{
SITUACIÓN CLIMA/ENERGÉTICA EN LOCALIDADES DE LA PROV. DE CÓRDOBA, EVALUACIÓN DE VIVIENDA TIPO; SIMULACIONES CON DIFERENTES ENVOLVENTES Y ORIENTACIONES, Y PROPUESTA DE ADECUACIÓN TÉRMICA, UTILIZANDO MUROS TROMBE U OTRAS FORMAS DE ENERGÍA SOLAR PASIVA
}

Eje 2: Tecnología para la construcción sustentable

Director: Arq. Guzmán Alberto Javier

Co-Director: Arq. Pilatti José Luis

Arq. Angulo Sergio

Arq. Piumetti José

Arq. Salinas Manuel

Pereyra Franco

Magister Túgel Julia

Olarte Armando

Facultad de Arquitectura Urbanismo y Diseño - Universidad Nacional de Córdoba, Argentina, arqalbertoguzman@hotmail.com

\section{RESUMEN}

La realidad actual a cerca del excesivo consumo y gasto energético que se produce en la etapa de uso de una vivienda, despertó nuestro interés, y enfoco nuestro trabajo hacia la búsqueda del cambio de paradigma en la forma de diseñar las mismas. Resolver un mal funcionamiento se resumirá en la aplicación de una serie de paliativos que generalmente serán a un alto costo. Las soluciones aplicadas seguramente desembocaran en grandes dificultades técnicas y restricciones en las posibilidades de intervención, dando lugar a problemáticas que impactan sobre la sociedad en su conjunto.

El desarrollo de nuestro trabajo se dividió en distintos ejes. Uno se enfocó hacia el tema de los "Grados Días" de calefacción y refrigeración, de gran importancia a la hora de determinar la cantidad de energía necesaria para lograr una situación de confort en el interior de nuestras viviendas. Para ello se tomó como datos de base los proporcionados por la Norma IRAM 11603, la cual determina los grados días de quince localizaciones de la Provincia de Córdoba, los que a nuestro entender, son escasos cuando se comienza a contemplar las diferentes características climáticas de nuestra provincia, y en especial los referidos a la zona oeste de nuestra provincia (zona serrana). Se trabajó sobre la búsqueda de datos de temperatura medias máximas y mínimas de alrededor de sesenta localizaciones distintas que se agregaron a las ya contempladas 
SITUACIÓN CLIMA/ENERGÉTICA EN LOCALIDADES DE LA PROV. DE CÓRDOBA, EVALUACIÓN DE VIVIENDA TIPO; SIMULACIONES CON DIFERENTES ENVOLVENTES Y ORIENTACIONES, Y PROPUESTA DE ADECUACIÓN TÉRMICA, UTILIZANDO MUROS TROMBE U OTRAS FORMAS DE ENERGÍA SOLAR PASIVA

por la Norma IRAM, las cuales fueron procesadas mediante una planilla electrónica elaborada por nuestro grupo de trabajo, obteniendo como resultado una mayor precisión en cuanto a esta información.

Por otro lado se trabajó en la simulación del comportamiento energético de una vivienda de las tipologías del "Plan Procrear", tomando como base las características constructivas de las mismas en función a las que ofrece dicho plan nacional. Para la confección de dicho trabajo se procesaron los datos en dos software específicos en el tema; por un lado ECOTEC (de características técnicas y graficas) y SIMEDIF (de características más analíticas). Se procedió a la verificación del comportamiento de la tipología tal cual fue ejecutada, y posteriormente modificada, reemplazando algunos elementos del diseño original por elementos pasivos para el aprovechamiento solar como es el caso de "muros Trombe-Mitchell". A partir de la incorporación de estos elementos pasivos, se realizó una nueva verificación del comportamiento energético de la tipología analizada. Este procedimiento, enlaza los datos obtenidos en un trabajo anterior realizado por nuestro grupo, relacionado con el estudio de este sistema pasivo de aprovechamiento de la energía solar.

\section{PALABRAS CLAVES: GRADOS DIAS - MURO TROMBE - ARQUITECTURA SUSTENTABLE}

\section{INTRODUCCION}

El consumo de energía que consumirá una vivienda en su etapa de uso, debe ser estudiada en su etapa de proyecto. Errores proyectuales se traducirán en la aplicación de una serie de paliativos de alto costo. Las soluciones aplicadas seguramente desembocaran en grandes dificultades técnicas y restricciones en las posibilidades de intervención, dando lugar a problemáticas que impactan sobre la sociedad en su conjunto; tales como un excesivo gasto energético , intensificando las problemáticas ambientales, agotamiento acelerado de los combustibles no renovables e impacto negativo sobre el balance comercial. En zonas sin acceso a la red de gas y sectores de la población menos pudientes la pobreza energética, entendida como la incapacidad de mantener la temperatura de las viviendas dentro de los rangos aconsejados por la OMS, impacta negativamente sobre el confort, y en casos extremos, sobre la salud de los habitantes. Desde nuestro punto de vista, creemos que la UNC y específicamente la FAUD y la FCEFyN con las Ingenierías vinculadas a la construcción de viviendas y edificios deben asumir el cambio de paradigma que implica pensar en el costo energético de funcionamiento de los edificios que nuestros profesionales diseñan y construyen. Nuestro objetivo se centró en la búsqueda de poder abordar el conocimiento de diversas tecnologías y técnicas para incorporar al proceso de proyecto de la arquitectura es que arribamos al concepto promovido desde hace ya muchos años por diversos autores. El presente trabajo consistió en evaluar los grados días de diversas localidades de la Prov. de Córdoba, aplicándolos a una tipología reconocida como las del plan "Procrear", estudiando y modificando sus envolventes y su orientación para lograr la calefacción pasiva, empleando principalmente los resultados obtenidos en el trabajo previo subsidiado por SeCyT (DESARROLLO, ENSAYO Y EVALUACIÓN DE MUROS COLECTORES / ACUMULADORES PASIVOS DE ENERGÍA SOLAR Y SU APROVECHAMIENTO PARA MEJORAR LAS CONDICIONES DE CONFORT TÉRMICO DE EDIFICIOS EN CLIMAS TEMPLADOS-SECOS. UN CASO PARTICULAR LA CDAD DE CBA Y CERCANÍAS) para su verificación en un caso real. 
Resumiendo, proponemos evaluar el déficit de energía en una tipología de vivienda y analizar la forma de reponer esa energía para mantenerla en el nivel térmico adecuado utilizando recursos pasivos de climatización como el muro Trombe ya estudiado previamente.

A nuestro entender, consideramos de gran importancia el presente trabajo de investigación, teniendo en cuenta las políticas de ahorro y eficiencia energética en general, promovidas por el Estado Nacional, específicamente en las temáticas de la construcción a través de diversos programas implementados por la Secretaria de Energía de la Nación. Nuestro Objetivo es lograr determinar parámetros o pautas de diseño concretas para las envolventes se busca comenzar a incorporar elementos pasivos de aprovechamiento de energías renovables a bajos costos, enfocados hacia las características de la geografía de la Provincia de Córdoba. Colaborando así con el cuidado del ambiente, a la eficiencia energética y a promover el cambio del paradigma energético en el cual está fundada la enseñanza y la práctica de la arquitectura actual.

\section{DESARROLLO}

\section{¿QUE SON LOS GRADOS DIAS?}

Desde épocas tan tempranas como en la segunda mitad del siglo XX se notó la necesidad de introducir un parámetro que, estando ligado a la temperatura ambiental, permitiera relacionar de manera práctica el sistema energía-clima, destacándose para ello el término grados-día [Thom, 1966]. En vista a establecer relaciones y efectuar pronósticos de consumo de energía para propósitos de calefacción de edificios y/o climatización de locales surge como importante el denominado como "método de los grados-día", requiere discernir entre los grados-día de calentamiento (GDC), y los grados-día de enfriamiento (GDE), tomando como criterio de comparación una llamada «temperatura base» (TB). Esta es una magnitud propia de cada país o región que define la temperatura de confort interior de la arquitectura. De esta manera la temperatura base no puede ser un valor constante para todo el planeta, ni aún para regiones de mediana magnitud y tiende a variar, sobre todo con la latitud. Tan es así, que por ejemplo, para el Reino Unido la TB que se usa es $15,5^{\circ} \mathrm{C}$ [Vesma, 2001], mientras que en los Estados Unidos se ha determinado que para los estados del Norte, esta es de $14{ }^{\circ} \mathrm{C}$, y para los del Sur, de $17{ }^{\circ} \mathrm{C}$; sin embargo, Sailor y Muñoz [1997] demostraron que para el estado de la Florida, la TB debe ser de $21^{\circ} \mathrm{C}$.

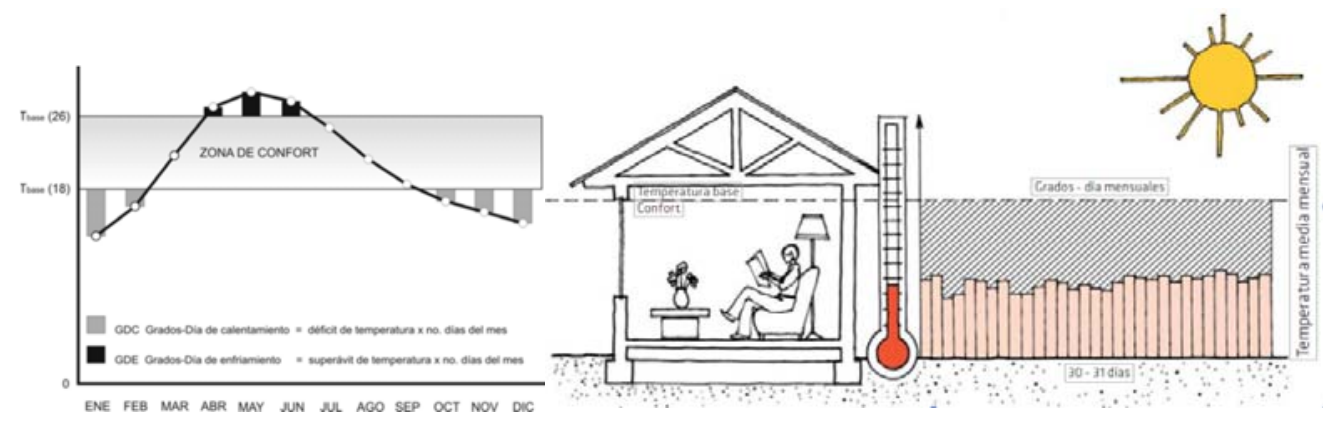

Esta temperatura base representa la temperatura en la cual debe existir un balance energético tal en el objeto de análisis (edificios residenciales, comerciales, industriales, etc.) en que no se requiera calefacción ni enfriamiento para obtener una sensación agradable para sus ocupantes, aunque esta no dependa íntegramente del parámetro temperatura, pero sí de factores relacionados con ella directa o indirectamente [UNEP/IES, 1998]. Los Grados-Día (también citado 
como Días-Grado) es un parámetro importante a considerar para la definición de las estrategias de diseño, los requerimientos de climatización (natural o artificial) y por lo tanto la demanda de energía de una edificación. Los Grados-Día se pueden definir como los requerimientos de calentamiento o enfriamiento (en grados centígrados o Kelvin1), necesarios para alcanzar la zona de confort, acumulados en un cierto período de tiempo mensuales, semanales y en algunos casos horarios).

La Norma IRAM 11549 (año 2002-4-12), en su capítulo de "aislamiento térmico de edificios", define a los "grados días de calefacción" como: Suma de las diferencias de temperaturas entre una temperatura base y la media diaria, para los días en que la media diaria es menor que la temperatura base en un periodo establecido (la norma IRAM 11603 indica los grados días anuales de calefacción con base. En forma resumida grados día son la suma de las diferencias entre la temperatura exterior y una de referencia (o base) en un periodo determinado. Nos permite estimar la demanda de energía para mantener el edificio en condiciones de confort en los distintos periodos del año, ya sea con calefacción o refrigeración, las que pueden ser aportadas por elementos pasivos de climatización.

Los Grados-Día se refieren al déficit o superávit acumulado de los 365 días del año2, es decir3:

Dónde:

$$
G D=\sum_{1}^{365}\left(T-T_{\text {baso }}\right)
$$

$G D=$ Grados-Día $\left({ }^{\circ} \mathrm{C}\right)$

Tbase $=$ Temperatura base (límite de referencia, inferior o superior de confort $\left({ }^{\circ} \mathrm{C}\right)$

$T=$ Temperatura media diaria $\left({ }^{\circ} \mathrm{C}\right)$

Aunque también puede analizarse de manera desagregada mes por mes:

$$
G D=\sum_{1}^{n}\left(T-T_{\text {base }}\right)
$$

$n=$ número de días del mes

2 C.f. Szokolay, Steven. (2004) Introduction to Architectural Science: The Basis of Sustainable Design Elsevier-Architectural Press. Great Britain. p. 32

Como punto de partida del presente trabajo de investigación, se analizaron diferentes autores relacionados con el tema Grados días.

FUENTES CONSULTADAS

GRADOS-DÍA EN ARQUITECTURA - ARQUITECTURA BIOCLIMATICA

Víctor Armando Fuentes Freixanet - Capitulo 8 - CONFORT TERMICO - UNIVERSIDAD AUTONOMA METROPOLITANA AZCAPOTZALCO - DIVISION DE CIENCIAS Y ARTES PARA EL DISEÑO

DEPARTAMENTO DEL MEDIO AMBIENTE 
REGIONALIZACION BIOCLIMATICA DE ARGENTINA MEDIANTE EL USO DE TECNICAS MULTIVARIADAS Y SIG - Jorge Daniel Czajkowski - Analía Gómez - Irene Martini - Yael Rosenfeld

TRABAJO INTEGRADOR FINAL - ADAPTACION DE UNA VIVIENDA PRO.CRE.AR A UNA VIVIENDA BIOCLIMATICA EN LA CIUDAD DE RAUCH, PROVINCIA DE BUENOS AIRES Tutora: Arq. Cecilia Corredera - Alumnas: Arq. Guillermina Rey - Arq. María Antonieta Sánchez

ESPECIALIZACION EN ARQUITECTURA Y HABITAT SUSTENTABLE. DIRECTOR DR. JORGE CZAJKOWSKI. - UNIVERSIDAD NACIONAL DE LA PLATA - FACULTAD DE ARQUITECTURA Y URB.

En función de que la Norma 11603, solamente toma/da los datos relacionados con los grados días de quince localidades de la Provincia de Córdoba, se decidió ampliar el espectro de localidades, a los fines de lograr mayor precisión y amplitud en el conocimiento de cómo es la Provincia de Córdoba en función de sus microclimas relativos. Con tal objeto se obtuvieron los datos temperatura y humedad relativa de 71 localidades. Algunos suministrados por el Servicio Meteorológico Nacional (homologados por el tiempo), y otros datos suministrados por el INTA y la Bolsa de Cereales de Córdoba. Dicha información se procesó tomando como referencias a herramientas y metodologías utilizadas por el programa "CEMATEM" DE GUILLERMO GONZALO (PROGRAMA PARA GRAFICAR LAS TEMPERATURAS). Para esto calcula las temperaturas horarias mensuales. Esto lo realiza ingresando las Temp. Max. Media mensuales, las Temp Min. Medias mensuales, de allí obtiene la diferencia de temperaturas medias mensuales y realiza la siguiente operación:

\section{DIFER.TEMPER. X COEF. + TEMP.MÍN.MED. = TEMPERATURAS HORARIAS}

Temp. Min. Media $+\Delta T$ (Dif. Entre le TMax y Min medias) X COEF (un coeficiente que distribuye en las 24 horas del día), generando así las temperaturas horarias.

La herramienta informática (fórmula para el cálculo y su correspondiente planilla Excel) utilizada por nuestro equipo de trabajo, fue modificada y ajustada a nuestras necesidades. Se toma como referencia también, el método GRADOS-DÍA EN ARQUITECTURA de Víctor A. Fuentes Freixanet. En la misma se puede observar la incorporación de datos de temperaturas y humedades relativas medias máximas y mínimas para una determinada localización. El procesamiento de estos

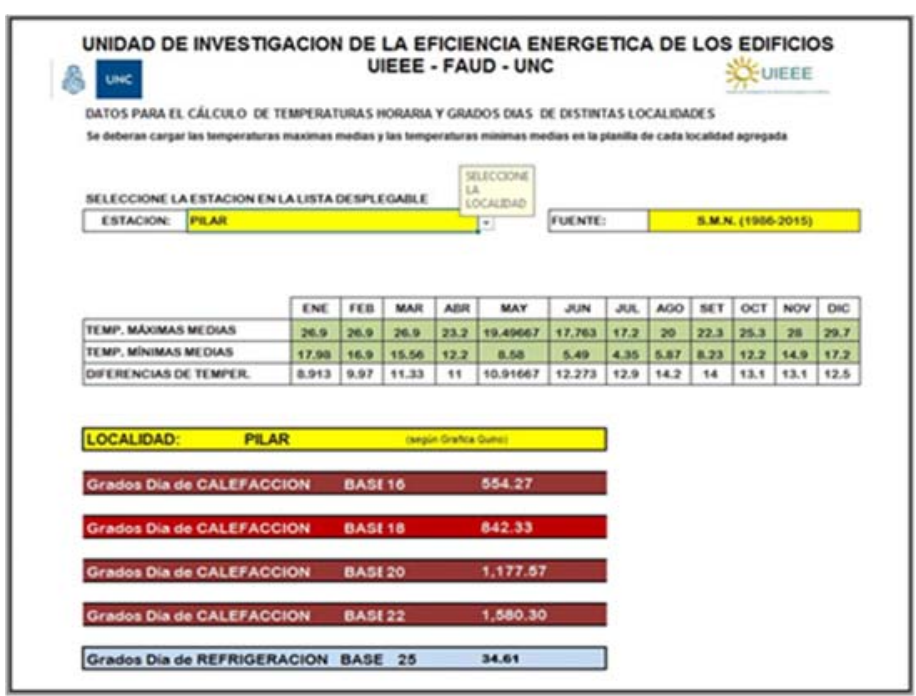
datos por la planilla electrónica nos arroja como resultado el cálculo de los grados días de calefacción (para una base de 16-18-20-22 grados) y refrigeración (a partir de $25^{\circ} \mathrm{C}$ ) para la localización analizada. 


\section{RESULTADOS OBTENIDOS DE GRADOS DIAS CALEFACCION POR LOCALIDADES}

\begin{tabular}{|r|l|r|}
\hline № & \multicolumn{1}{|c|}{ LOCALIDAD } & GD -calef. \\
\hline 1 & ALTA GRACIA & 1010.83 \\
\hline 2 & ANISACATE & 952.42 \\
\hline 3 & ARROYITO & 912.73 \\
\hline 4 & ASCOCHINGA & 1489.18 \\
\hline 5 & BELLVILLE & 964.23 \\
\hline 6 & BIALET MASSE & 1059.11 \\
\hline 7 & BOUWER & 986.57 \\
\hline 8 & BRINKMAN & 804.6 \\
\hline 9 & CAPILLA DEL MONTE & 1420.74 \\
\hline 10 & CHARBONIER & 1295.2 \\
\hline 11 & CHUÑA HUASI & 806.02 \\
\hline 12 & COLONIA CAROYA & 1185.23 \\
\hline 13 & CORDOBA AEROPUERTO & 864.67 \\
\hline 14 & CORDOBA OBSERVATORIO & 740.16 \\
\hline 15 & COSQUIN & 1111.39 \\
\hline 16 & CUESTA BLANCA & 1119.66 \\
\hline 17 & DEAN FUNES & 1070.16 \\
\hline 18 & DESPEÑADEROS & 977.03 \\
\hline 19 & DIQUE CRUZ DEL EJE & 516.42 \\
\hline 20 & DIQUE LA VIÑA & 969.63 \\
\hline 21 & DIQUE PISCO HUASI & 885.21 \\
\hline 22 & EMBALSE & 848.9 \\
\hline 23 & FALDA DEL CARMEN & 1059.64 \\
\hline 24 & FREYRE & 861.46 \\
\hline 25 & HUANCHILLA & 998.3 \\
\hline 26 & HUARTE GRANDE & 1281.34 \\
\hline 27 & ISCHILIN & 1335.04 \\
\hline 28 & ISLA VERDE & 1069.21 \\
\hline 29 & JESUS MARIA & 1247.34 \\
\hline 30 & JOSE DE LA QUINTANA & 993.51 \\
\hline 31 & LA CALERA & 966.05 \\
\hline 32 & LA CARLOTA & \\
\hline 33 & LA CUMBRE & 141.22 \\
\hline 34 & LA CUMBRECITA \\
\hline 35 & LABOULAYE & \\
\hline 36 & LAS ACEQUIAS & \\
\hline
\end{tabular}

\begin{tabular}{|l|l|r|}
\hline No & \multicolumn{1}{|c|}{ LOCALIDAD } & GD -calef. \\
\hline 37 & LAS VARILLAS & 979.16 \\
\hline 38 & LUCIO V. MANCILLA & 545.92 \\
\hline 39 & MARCOS JUAREZ & 1002.9 \\
\hline 40 & MINA CLAVERO & 1167.01 \\
\hline 41 & MIRAMAR & 625.13 \\
\hline 42 & MONTE MAIZ & 1077.58 \\
\hline 43 & MORTEROS & 789.48 \\
\hline 44 & OBISPO TREJO & 801.39 \\
\hline 45 & PILAR & 842.33 \\
\hline 46 & QUILINO & 681.97 \\
\hline 47 & RAFAEL GARCIA & 970.92 \\
\hline 48 & RIO CEBALLOS & 1317.56 \\
\hline 49 & RIO CUARTO & 988.29 \\
\hline 50 & SALSACATE & 1135.48 \\
\hline 51 & SAN AGUSTIN & 1069.46 \\
\hline 52 & SAN CLEMENTE & 1333.73 \\
\hline 53 & SAN FRANCISCO & 914.27 \\
\hline 54 & SAN FRANCISCO DEL CHAÑAR & 992.12 \\
\hline 55 & SAN JAVIER & 1125.85 \\
\hline 56 & SAN JOSE DE LA SALINA & 547.93 \\
\hline 57 & SAN PEDRO & 1435.42 \\
\hline 58 & SANTA CATALINA & 1049.72 \\
\hline 59 & SANTA ROSA DE CALAMUCHITA & 1064.31 \\
\hline 60 & SANTA ROSA DE RIO PRIMERO & 895.51 \\
\hline 61 & SEBASTIAN ELCANO & 776.66 \\
\hline 62 & VILLA DE MARIA DE RIO SECO & 1149.64 \\
\hline 63 & VICUÑA MAKENA & 1040.51 \\
\hline 64 & VILLA ALPINA & 1696.77 \\
\hline 65 & VILLA CARLOS PAZ & 1037.36 \\
\hline 66 & VILLA DE SOTO & 820.82 \\
\hline 67 & VILLA TOTORAL & 1174.06 \\
\hline 68 & VILLA DOLORES & 728.65 \\
\hline 69 & VILLA ICHO CRUZ & 961.15 \\
\hline 70 & VILLA LA BOLSA & \\
\hline 71 & VILLA MARIA & \\
\hline 72 & & \\
\hline
\end{tabular}

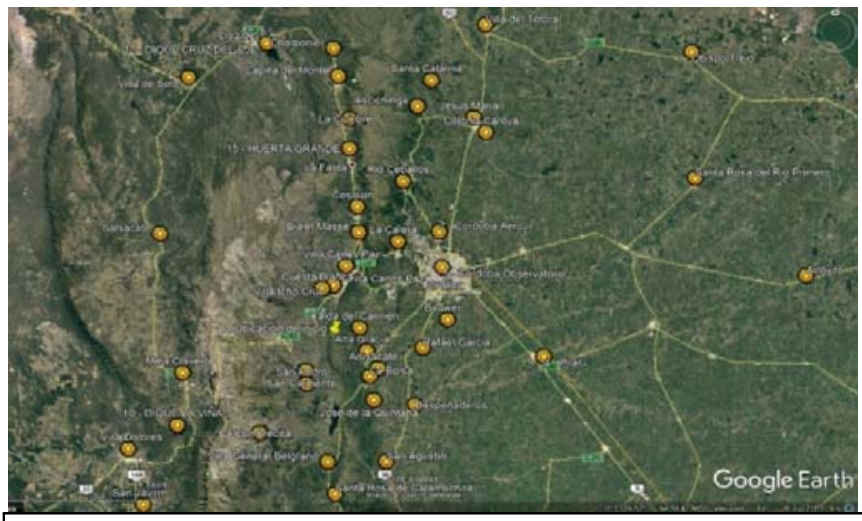

MAPA DE UBICACIÓN DE LOCALIDADES ANALIZADAS

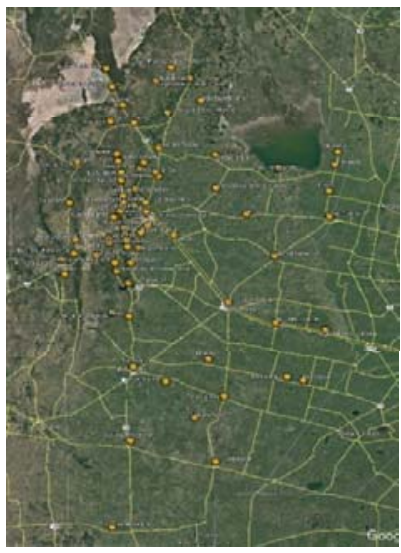

SIMULACIÓN DE UNA VIVIENDA TIPO: Se tomó para este trabajo una tipología del Plan PROCREAR, a los fines de evaluar la performance térmica de este tipo de desarrollos proyectuales elaborados por el Estado Nacional. 


\section{DESCRIPCION DEL PROTOTIPO ANALIZADO - Vivienda PROCREAR}

\begin{tabular}{|l|l|}
\hline TIPOLOGIA & Vivienda unifamiliar \\
\hline UBICACION & Comuna de Anisacate - Provincia Córdoba \\
\hline SUPREFICIE CUBIERTA & $75,00 \mathrm{~m} 2$ (aproximadamente) \\
\hline SISTEMA CONSTRUCTIVO & Sistema tradicional racionalizado. \\
& Envolvente norte: Ladrillo macizo común $30 \mathrm{~cm}$. \\
& Envolventes este-oeste-sur: muro doble hoja ladrillo macizo común \\
& exterior y bloque cerámico portante $12 \mathrm{~cm}$. Interior. \\
& Envolvente superior: Estructura madera, aislaciones y chapa. \\
& Losa maciza sobre dormitorio, con cubierta techo tradicional. \\
& Aberturas: Vidrios simples. Perfiles "T"de acero y otras de aluminio. \\
& Piso: cerámico sobre carpeta cementicia y contrapiso \\
\hline
\end{tabular}

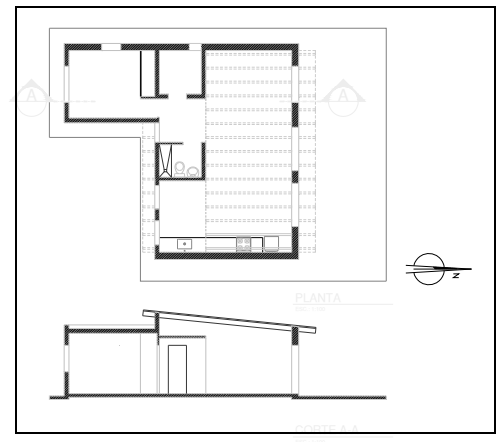

$$
\begin{aligned}
& \text { FOTO 1: Esquina fachadas norte-oeste } \\
& \text { FOTO 2: Interior estar } \\
& \text { FOTO 3: Esquina fachadas este-norte } \\
& \text { FOTO 4: Esquina fachadas sur-este } \\
& \text { FOTO 5: Fachada oeste dormitorio }
\end{aligned}
$$
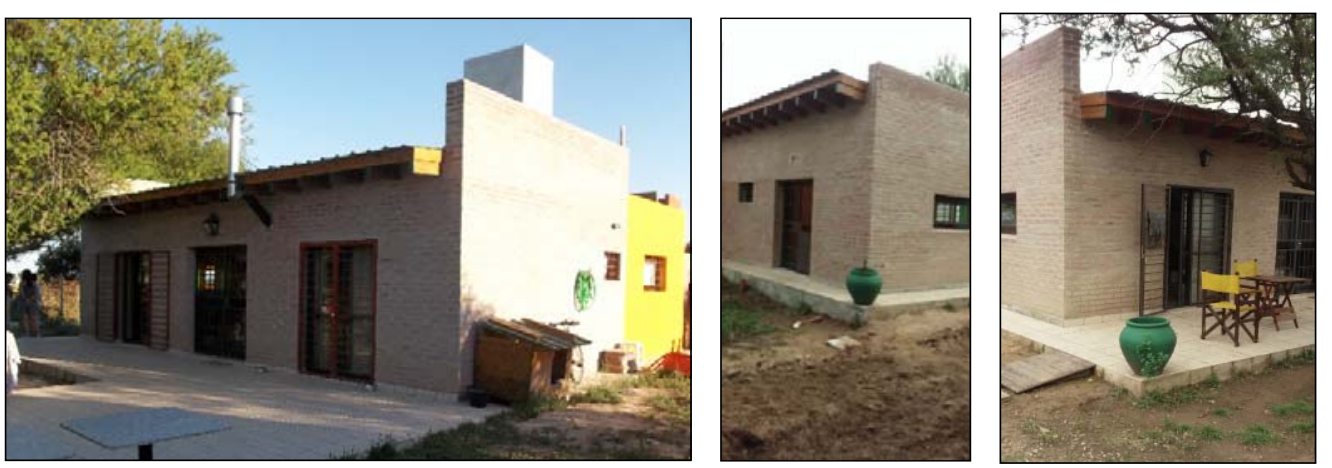

\section{COMPARACIÓN DE CONDICIONES SIMULADAS Y MEDIDAS, PROGRAMA DE SIMULACIÓN.}

Tipología de vivienda en su diseño inicial y con la simulación de incorporación de muro Trombe Mitchell en su fachada norte en el mes de: JUNIO
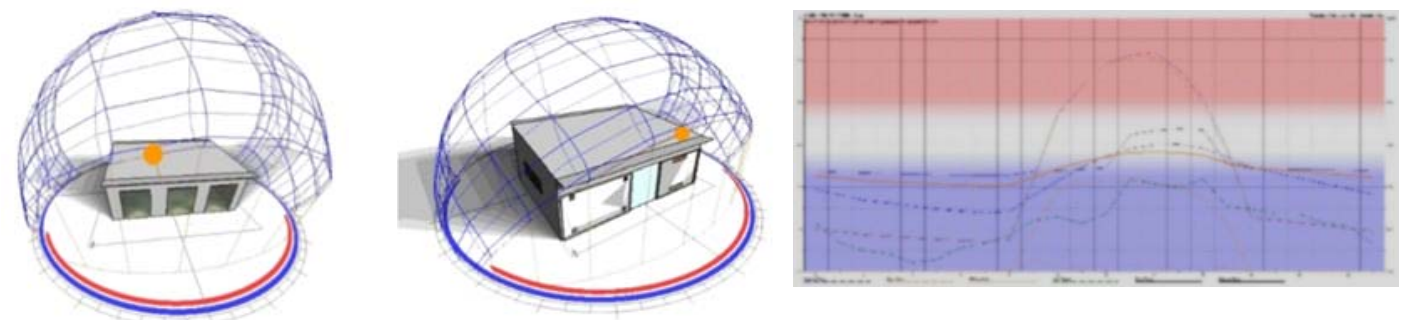

Nótese como la radiación solar incide plenamente en toda la cara NORTE y las curvas de temperatura en los muros Trombe ascienden a valores como los registrados en el ensayo real desarrollado en los años 2015 en el proyecto previo. 
SEPTIEMBRE: Las temperaturas en los muros Trombe disminuyen notablemente, así como la radiación solar incidente en estos.
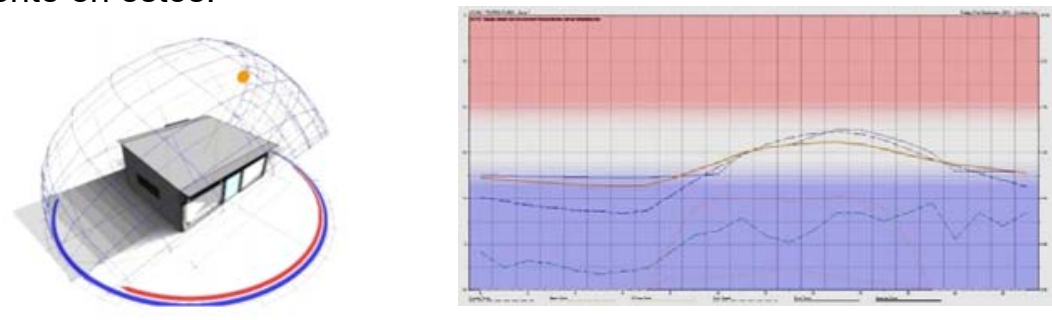

\section{DICIEMBRE}
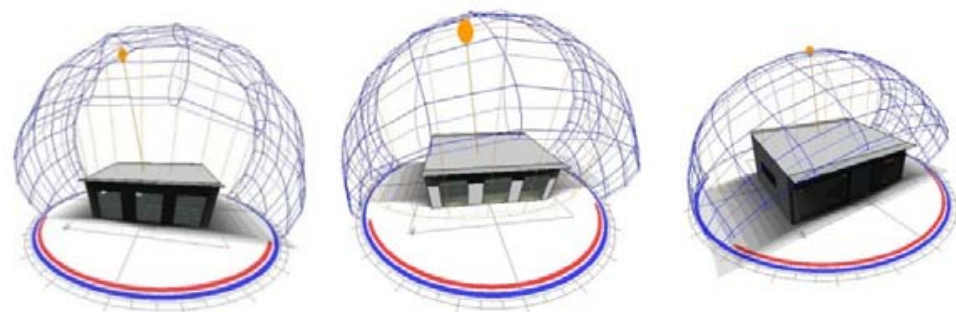

En esta época del año la radiación solar directa recibida es nula.

COMPORTAMIENTO DE LA TIPOLOGIA SIN MURO TROMBE ANUAL- $1^{\circ}$ DE enero al 31 de diciembre COMPORTAMIENTO DE LA TIPOLOGIA CON MURO TROMBE- $1^{\circ}$ DE enero al 31 de
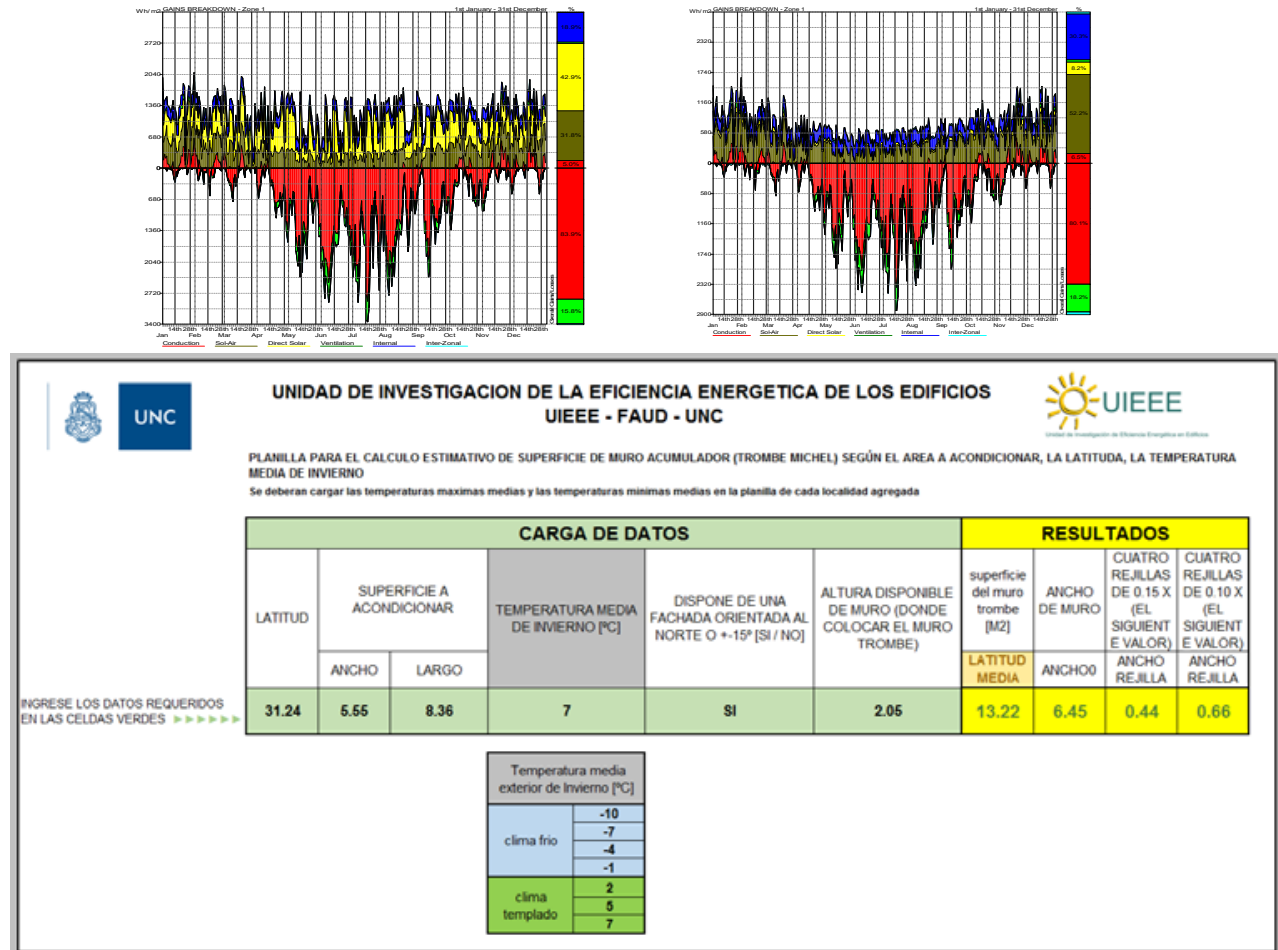

En las dos graficas previas, se muestran de manera analíticas las pérdidas y ganancias de calor notándose la mayor ganancia por conducción (sol-air) en el caso donde se trabajó con el muro trombe. Esto no se manifiesta claramente al analizar las temperaturas interiores en el ambiente donde se debería elevar la temperatura (estar de la vivienda). Luego de numerosas 
averiguaciones y correcciones del modelo simulado, hemos detectado usuarios del sofware "Ecotect" con experiencias similares en simulaciones de este tipo de elementos pasivos de acondicionamiento térmico pasivo. La superficie de muro trombe simulada, responde al resultado obtenido en el proyecto SeCyT mencionado recientemente, del cual se generó una planilla de estimación de dimensiones de los muros Trombe en función de parámetros determinados por distintos autores y agregándole la ganancia real para una latitud como la nuestra, determinando un rendimiento y la estimación de la energía ganada en el ambiente interior.

Se obtuvo del cálculo previo, la necesidad de colocar $13.22 \mathrm{~m} 2$ de muro Trombe los que fueron distribuidos en dos paneles de 2.00 metros de alto por 2.40 y 3.00 metros de ancho respectivamente. En función de la energía recibida por

relacion entre energia recibida y entregado al interior

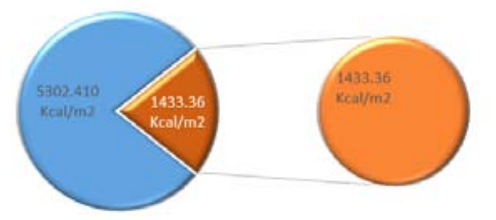
estos, se eleva la temperatura de la cara exterior del muro Trombe, ingresando al interior por conducción a través de la mampostería que lo constituye, elevando la temperatura interior, aplanando la onda térmica, es decir: cuando ingresa el calor acumulado en la cara exterior y se reirradia hacia el interior debido al retardo térmico generado en el proceso de conducción (aproximadamente 6:30 hs), en el exterior descendió notablemente la temperatura ambiente. Pese a lo que

sucede en el exterior la temperatura interior se mantiene estable por el aporte del muro Trombe. La cantidad de energía recibida por m2 de muro en un día tipo del invierno de nuestra región es de $1433.36 \mathrm{Kcal} / \mathrm{m} 2 x d i a$, por lo tanto nuestra vivienda en estudio recibirá un aporte térmico de $18949 \mathrm{kcal} / \mathrm{día}$. Ni más ni menos que un calefactor a gas convencional de $3000 \mathrm{kcal} / \mathrm{h}$, con un rendimiento de $100 \%$, funcionando durante 6,20 horas. Hemos combinado el uso de dos programas de simulación: Simedif y EcotectSimedif no posee una interfase gráfica lo que hace más trabajosa la "construcción" de la vivienda o mejor dicho de su modelo físico matemático. La fortaleza del programa es la mayor precisión comparada con Ecotect debido al modelo matemático que utiliza que tiene en cuenta conductividad, calor específico, densidad y espesor de los materiales por un lado y por otros coeficientes de absorción, convección, descarga entre otros.

\section{CONCLUSIONES}

Con relación a los estudios acerca del comportamiento de los muros trombe, hemos logrado establecer que del $100 \%$ del muro con orientación Norte, casi un $25 \%$ ingresa al interior, lo cual equivale a aprox. $1.433 \mathrm{Kcal} / \mathrm{m} 2$. En función de estos datos se elabora una planilla electrónica para el cálculo del área necesaria de muro Trombe. En ella hemos logrado, en función de una serie de datos (latitud del lugar, superficies a acondicionar, temperaturas medias exterior de invierno); determinar la superficie necesaria que debe tener el/los muros Trombe que vamos a incorporar a nuestra arquitectura. Con relación al estudio realizado sobre los grados días, hemos logrado ampliar el espectro de datos proporcionados por la Norma IRAM 11603 (1996), incorporando un número mayor de localidades en base a las cuales se podrán trazar líneas de isobaras con relación a los grados días, lo que nos permitirá un mayor grado de precisión a la hora de diseñar la arquitectura, teniendo en cuenta esta variable, en pos de la construcción de un hábitat más confortable y sustentable. 


\section{BIBLIOGRAFIA}

Normas IRAM 11601/96; 11603/96; 11604 / 2001; 11605/96; 11900/2010

Passive Solar Design. (December 2000). Work Performed by the NAHB Research Center, Southface Energy Institute, and Oak Ridge National Laboratory. Washington, D.C.:U.S. Department of Energy-

Passive Solar Energy. (2006). B. Anderson, M. Wells.

Kachadorian, J. (1977). The Passive Solar House. White River Jct-, VT:Chelsea Green Publishing Co.

Van Dresser, P. (1996). Passive Solar House Basics. Santa Fe, NM: Ancient City Press.

Gonzalo, Guillermo y Otros. Evaluación energética en edificios. Centro de Estudios Energía y Medio Ambiente (CEEMA), Inst. de Acondicionamiento Ambiental-FAU-UNT-

F.Javier Neila González. Arquitectura bioclimática en un entorno sostenible. Edit. MunillaLeria.(2004)

Investigac Programa EEE (Eficiencia Energética en Edificios),FAUDI,UNC: 2005/2011

Clima de Córdoba. - Evaluación del confort en la arquitectura- Sistemas de evaluación del asoleamiento. - Sistemas de evaluación de la iluminación.- Sistemas de evaluación de la transmitancia térmica y la condensación en muros.- Sistemas de evaluación de ventilación.Sistemas de evaluación integrales del comportamiento energético ambientales de los edificios.

Crosbie, M.J., Ed (1997). The Passive Solar Design and Construction Handbook. New York: John Wiley \& Sons, Inc.

Onishi J, Soeda H, Mizuno M; Numerical study on a low energy architecture based upon distributed heat storage system. Renewable Energy 2001; V 22:61

DOMANCIC HERRERA, Pedro José. Diseño de un Sistema de Calefacción Solar para un Edificio Público. Santiago: Universidad de Chile, Departamento de Ingeniería Mecánica, 2008. Memoria (Ingeniero Civil) Universidad de Chile.

Bienvenido a la casa solar pasiva; http://www.benyei.org/Solar/trombe.htm

Yunus A. Cengel, A. J. Ghajar, Transferencia de Calor y Masa, 4² Edición,McGraw-Hill,2011.

Hoy-Yen Shan, Saffa B. Riffat, Jie Zhu; Review of Passive solar heating and cooling technologies; Renewable and Sustainable Energy Reviews 2010; V 14:781-789

Principios fundamentales de paredes Trombe y Chinemenas Solares; http://sallavor.org/resources/Muro\%2Btrombe\%2By\%2Bchimeneas\%2Bsolare.pdf

Jibao Shen, Stéphanne Lassue, Lawrent Zalewski, Dezhong Huang; Numerical study on thermal behavior of clasical or composite Trombe solar walls; Energy and buildings 2007; V39:962-974 